\title{
Caractérisation de quelques écotypes performants de sorghos Burkinabés
}

\author{
Gapili NAOURA ${ }^{1,2, *}$, Baloua NEBIE ${ }^{3}$, Romaric Kiswendsida NANEMA ${ }^{2}$, \\ Pauline BATIONO KANDO ${ }^{2}$, Ernest Renan TRAORE ${ }^{2}$, Mahamadou SAWADOGO ${ }^{2}$ \\ et Jean-Didier ZONGO ${ }^{2}$ \\ ${ }^{I}$ Institut Tchadien de Recherche Agronomique pour le Développement (ITRAD), BP 5400 Ndjamena, Tchad. \\ ${ }^{2}$ Equipe Génétique et Amélioration des Plantes, Ecole Doctorale Sciences et Technologies, \\ Université de Ouagadougou 03, BP 7021 Ouagadougou 03, Burkina Faso. \\ ${ }^{3}$ ICRISAT-Bamako, BP 320 Bamako, Mali. \\ *Auteur correspondant; E-mail : gap_pablo@yahoo.fr; Tel :(+226) 672175 19/(+235) 63381093
}

\section{RESUME}

Cinquante-huit (58) écotypes de sorgho ont été sélectionnés dans la collection de sorghos de l'Université de Ouagadougou et caractérisés dans le cadre de l'évaluation de leur performance. Le choix des écotypes a porté sur leur productivité et les qualités liées à leur appréciation par les paysans. La caractérisation agronomique a révélé l'existence d'une très grande variabilité autour des caractères liés à la productivité. L'analyse de la matrice de corrélation a révélé des associations positives entre les caractères de production et ceux de rusticité telle l'aptitude au tallage. La comparaison réalisée avec l'évaluation de 1991 a montré des différences dont la plus importante est celle liée au rendement potentiel. L'identification des écotypes performants et des géniteurs de caractères a permis de ressortir quelques écotypes dont les qualités liées tant à la production qu'à la rusticité sont des atouts autour de quoi, l'espoir peut être fondé dans l'accroissement des productions et l'amélioration de sorghos du Burkina Faso.

(C) 2014 International Formulae Group. All rights reserved.

Mots clés : Sorghos performants, géniteurs de caractères, variabilité, productivité.

\section{INTRODUCTION}

Le sorgho est la principale céréale cultivée au Burkina Faso avec une production annuelle d'environ 1,2 million de tonnes par an, occupant environ $54 \%$ des superficies céréalières (Brocke et al., 2002). La moyenne des rendements grain est relativement faible, autour de $800 \mathrm{~kg} / \mathrm{ha}$, avec cependant des variations importantes suivant les zones de production de $1050 \mathrm{~kg} / \mathrm{ha}$ dans la région Ouest contre $532 \mathrm{~kg} / \mathrm{ha}$ dans la région du Sahel (Trouche et al., 2001).
Le Burkina Faso est considéré comme un centre secondaire de diversité des sorghos cultivés (Chantereau et al., 1997). Les variétés locales qui y sont cultivées sont adaptées aux multiples conditions écologiques et aux divers objectifs de production des agriculteurs (Zongo, 1991). Au cours des trente dernières années, la production du sorgho a presque doublé mais celle-ci est surtout liée à l'augmentation des superficies (Hanak-Freud, 2000).

Malgré l'existence de programmes de recherches sur l'amélioration de sorghos 
depuis les années soixante, le taux d'adoption des variétés sélectionnées est resté très faible, représentant suivant les régions 2 à $5 \%$ des surfaces cultivées (Trouche et al., 2001). Cette faible adoption des variétés améliorées est due aux difficultés d'adaptation de celles-ci dans ces milieux très fluctuants. Contrairement aux variétés améliorées, les variétés traditionnelles possèdent des caractéristiques d'adaptations. Malheureusement, le sorgho subit une érosion génétique due à la grande variabilité interannuelle des pluies, la réduction de la durée des pluies, la dégradation des sols et la compétition avec d'autres cultures, notamment les cultures de rente (Brocke et al., 2002). Des travaux de collection et de caractérisation de la variabilité agromorphologique et biochimique ont été réalisés au Laboratoire de Génétique et de Biotechnologie Végétales de l'Université de Ouagadougou sur l'étendue du territoire entre 1984 et 1987 (Zongo, 1991). Des études récentes ont montré une importante variabilité génétique du sorgho au Burkina Faso (Nebié et al., 2013; Barro/Kondombo, 2010) Une importante variabilité a été mise en évidence et des écotypes performants sur le plan productivité ont été identifiés.

L'objectif de la présente étude est de valoriser le germoplasme de l'Université de Ouagadougou en partant par l'identification dans celle-ci des meilleurs écotypes locaux pour les caractéristiques recherchées pour une observation plus poussée en vue de mieux cerner leur performances et d'identifier des géniteurs potentiels pour des futurs programmes d'amélioration.

\section{MATERIEL ET METHODES Matériel végétal}

Les travaux ont porté sur 58 écotypes issus de la collection nationale constituée par le Laboratoire de Génétique et de Biotechnologie Végétales de l'Université de Ouagadougou. Ces écotypes ont été les plus performants lors de l'évaluation agromorphologique réalisée en 1987, 1988
1989. Le Tableau 1 montre la répartition des écotypes selon leur localité d'origine.

\section{Dispositif expérimental et techniques culturales}

Les essais ont été réalisés à la station de recherche de l'Université de Ouagadougou situé à Gampèla, Burkina Faso $\left(12^{\circ} 25^{\prime} \mathrm{N}\right.$ et $\left.1^{\circ} 12^{\prime} \mathrm{W}\right)$, sur un sol argilo sableux, avec une pluviométrie de 865,9 et une température moyenne de $28,54{ }^{\circ} \mathrm{C}$.

La parcelle expérimentale a été préalablement défrichée et labourée au tracteur. Un dispositif en blocs complètement randomisés avec trois répétitions a été utilisé. Chaque bloc est divisé en deux sous-blocs séparés par une allée de $2 \mathrm{~m}$ avec chacun une longueur de $24 \mathrm{~m}$ et 31 lignes. Chaque ligne mesurant $6 \mathrm{~m}$ de long. L'interligne a été de $0,8 \mathrm{~m}$ et l'écartement de $0,4 \mathrm{~m}$. Chaque bloc est représenté au total par une longueur de 46 $\mathrm{m}$ sur une largeur de $6 \mathrm{~m}$ avec 62 lignes. Les allées entre les blocs ont été de $02 \mathrm{~m}$. L'essai est ainsi réalisé sur un dispositif de $46 \mathrm{~m}$ de long sur $22 \mathrm{~m}$ de large, soit une superficie de $1112 \mathrm{~m}^{2}$.

Les semis ont été effectués le 23/07/2008 sur un sol humide. Chaque écotype est semé sur une ligne, en raison de 16 poquets par ligne. Le démariage à un plant a été réalisé le 12/08/2008. Il est accompagné d'un sarclage et d'un apport d'engrais NPK (15-15-15) à la dose de $100 \mathrm{~kg} / \mathrm{ha}$. Puis un deuxième sarclage a été réalisé deux semaines après le premier. Un apport d'urée à la dose de $40 \mathrm{~kg} / \mathrm{ha}$ suivi d'un buttage des plants a été effectué 50 jours après semis, au stade montaison.

\section{Variables observées}

Deux catégories de caractères ont été étudiées. Il s'agit de caractères qualitatifs et quantitatifs.

Douze variables qualitatives ont été observées. Il s'agit de : couleur de la plantule (CPL), couleur de la graine (CGR), couleur des glumes (CGL), aristation (ART), type botanique (TBO), compacité de la panicule (CPA), type de pédoncule (TPD), pilosité de la glume (PGL), lustre de la graine (LGR), couverture de la graine $(\mathrm{CVG})$ et l'exertion (EXT). 
Seize variables quantitatives ont été mesurées. Il s'agit de: date de gonflement (GFL), nombre de jours à l'épiaison (NJE) et à la floraison (NJF), longueur (LOF) et largeur (LAF) de la troisième feuille sous-paniculaire, hauteur de la plante à maturité (HPM), longueur (LOP) et largeur (LAP) de la panicule, poids des grains de la panicule principale (GPA) et celui de la plante entière (GPL), poids de cent grains (PCG), nombre d'entre-nœuds (NEN), diamètre de la tige (DIT), tallage végétatif (TAV) et utile (TAI) et vitrosité (VIT).

\section{Analyse des données}

L'analyse des variances (ANOVA) réalisée sur les variables quantitatives a visé à évaluer le niveau de diversité du matériel végétal. Le coefficient de variation $(\mathrm{CV})$ a permis d'apprécier les niveaux de variation des moyennes observées entre les écotypes pour chaque caractère. Les corrélations ont permis de vérifier les liaisons deux à deux entre variables. L'ACP a permis, à partir de la corrélation des matrices de variables originelles, d'extraire un petit nombre de combinaisons linéaires non corrélées entre elles. Le regroupement entre caractères a été réalisé par l'analyse en composantes multiples (ACM).

Les logiciels XLSTAT-Pro 7.1 et Excel ont été utilisés pour les différentes analyses.

\section{RESULTATS}

\section{Caractères qualitatifs}

Le Tableau 2 donne les résultats de la notation des variantes pour les caractères qualitatifs. On remarque au niveau des races une prédominance des guinea avec une proportion de $77,08 \%$

Pour la couleur de glumes, les écotypes à glumes noires ont été majoritaires avec une proportion de 70,8\% de l'ensemble des écotypes. Pour le caractère vitrosité, les accessions à cornés (plutôt corné et corné) ont représenté $43,8 \%$ et corné à $50 \%$ avec $31,2 \%$. Les écotypes à grains blancs ont représenté $72,9 \%$ de l'ensemble des écotypes. Les arêtes sont présentes chez $83,33 \%$ des écotypes. Les panicules en cross et la présence de poils au niveau de glumes sont rares avec chacune une proportion de $2,13 \%$.

\section{Caractères quantitatifs}

Le Tableau 3 donne les résultats des performances moyennes des écotypes. Pour la plupart des caractères étudiés, les écart-types sont très faibles. Cependant le poids des grains de la plante entière (GPL) et le poids des grains de la panicule principale (GPA) ont des écart-types respectivement 16,19 et 11,29. Aussi, on note des coefficients de variation très élevés pour le tallage utile (TAI) et le tallage végétatif (TAV) qui sont respectivement de $75 \%$ et de $62 \%$.

L'analyse de variance montre l'existence des différences significatives au sein de l'échantillon pour la plupart des caractères sauf pour le poids de cent grains (PCG) et le nombre de panicule par poquet (NPP).

La matrice de corrélation (Tableau 4) donne des corrélations importantes entre les caractères de production. Le poids de grains de la plante entière (GPL) est fortement et positivement corrélé au poids des grains de la panicule principale (GPA). Le rendement paniculaire (GPA) est négativement corrélé au tallage utile (TAI), au nombre d'entre-nœuds (NEN), au cycle (NJF) et au nombre de talles basales (NTB). Le cycle (NJF) est fortement et positivement corrélé au nombre d'entrenœuds (NEN), au diamètre de la tige (DIT) et aussi corrélé positivement à la hauteur de la plante (HPM), au tallage utile (TAI), à la largeur de la panicule (LAP) et à la longueur de la panicule (LOP). La longueur de la panicule (LOP) est positivement corrélée avec la hauteur de la plante (HPM), la longueur de la feuille (LOF) et le cycle (NJF).

L'ACM réalisée (Figure 1) nous donne un premier axe avec 21,56\% d'inertie, qui d'une part oppose la vitrosité totale (VIT_tle) $\mathrm{au}$ poids de cent grains forts (PCG_frt) et d'autre part associe la vitrosité totale (VIT_tle) et la vitrosité forte (VIT_frt) au poids de cent grains faibles (PCG_fbl); puis un second axe avec $16,67 \%$ d'inertie qui 
associe le poids de cent grains forts (PCG_frt) à la vitrosité nulle (VIT_nul).

L'ACP réalisée avec l'évaluation de 2008 en supplément par rapport à celle de 1991 avec les mêmes écotypes (Figure 2) nous donne : un premier axe avec 39,24\% d'inertie, déterminé par le poids de cent grains, un deuxième axe avec $34,81 \%$ d'inertie, caractérisé par le poids des grains de la plante et un troisième axe qui est déterminé par le cycle semi-floraison. Les écotypes dans l'évaluation de 1991 se regroupent et présentent de meilleurs rendements par plante par rapport aux mêmes écotypes dans l'évaluation 2008. Par contre, les écotypes dans l'évaluation de 2008 portent de plus grandes valeurs pour le poids de cents grains par rapport aux mêmes écotypes dans l'évaluation 1991.
L'identification des géniteurs de caractères nous a conduit à retenir des écotypes pour les caractères suivants :

- Les sorghos les plus productifs sont ceux qui possèdent les meilleurs rendements par plante et les meilleurs rendements potentiels (écotype 31, suivi de 454, puis 195 et 5);

- Les géniteurs des caractères pour la taille des sorghos sont choisis parmi les sorghos les plus productifs. Ils sont généralement de petites tailles (écotype 285, suivi de 195 et de 454) ;

- Les géniteurs de caractères pour la grosseur des grains sont recherchés parmi les sorghos les plus productifs (écotype 37, suivi de 34 et de 3 ) ;

Les sorghos géniteurs pour la vitrosité sont représentés par deux écotypes. L'un avec une vitrosité totale (écotype 95) et l'autre une vitrosité forte (écotype 454).

Tableau 1 : Répartition des écotypes selon leur localité d'origine.

\begin{tabular}{lc}
\hline Nombre d'écotypes & Localité d'origine \\
\hline 25 & Sahel \\
06 & région de l'Est \\
09 & plateau Central Sud \\
15 & région du Nord-Ouest \\
03 & région de l'Ouest \\
\hline
\end{tabular}

Tableau 3 : Performance des écotypes de sorghos de la collection du Burkina Faso.

\begin{tabular}{lcccccc}
\hline Caractère & MIN. & MAX. & MOY, & ET & CV & F \\
\hline NJF & 62,00 & 80 & 68,56 & 4,47 & $7 \%$ & $* *$ \\
LOF cm) & 67,17 & 84,33 & 77,26 & 4,15 & $5 \%$ & $* *$ \\
LAF $(\mathrm{cm})$ & 6,80 & 16,78 & 8,20 & 1,45 & $18 \%$ & $*$ \\
HPM $(\mathrm{cm})$ & 170,67 & 395,75 & 320,77 & 0,47 & $15 \%$ & $* *$ \\
LOP $(\mathrm{cm})$ & 25,42 & 48,92 & 38,57 & 5,43 & $14 \%$ & $* *$ \\
LAP $(\mathrm{cm})$ & 8,08 & 15,42 & 11,35 & 1,61 & $14 \%$ & $* *$ \\
GPA $(\mathrm{g})$ & 23,02 & 75,55 & 55,98 & 11,29 & $20 \%$ & $* *$ \\
GPL $(\mathrm{g})$ & 37,86 & 104,25 & 67,32 & 16,19 & $24 \%$ & $* *$ \\
PCG $(\mathrm{g})$ & 1,59 & 4,03 & 2,27 & 0,38 & $17 \%$ & NS \\
DTI $(\mathrm{cm})$ & 1,58 & 2,28 & 1,89 & 0,18 & $9 \%$ & $* *$ \\
NEN & 7,25 & 12,50 & 8,90 & 1,22 & $14 \%$ & $* *$ \\
TAI & 0,07 & 2,07 & 0,49 & 0,37 & $75 \%$ & $* *$ \\
\hline \multicolumn{7}{r}{ *significatif, **hautement significatif, NS: non significatif, NJF : nombre de jours à la floraison, LOF : longueur de la } \\
feuille, LAF : largeur de la feuille, HPM : hauteur de la plante à maturite, LOP : longueur de la panicule, LAP : largeur \\
de la panicule, GPA : poids des grains de la panicule principale, GPL : poids des grains de la plante entière, PCG : poids \\
de cent grains, DTI : diamètre de la tige, NEN : nombre d'entre-nœuds, TAI : tallage utile.
\end{tabular}


Tableau 2 : Valeurs moyennes de caractères quantitatives et paramètres statistiques associés.

\begin{tabular}{lcc}
\hline Caractères analysés & Modalités & Fréquences (\%) \\
\hline \multirow{2}{*}{ Types botaniques (TBO) } & Guinea & 77,08 \\
& Durra & 14,58 \\
& Bicolor & 4,17 \\
& Caudatum & 4,17 \\
\hline \multirow{2}{*}{ Couleur des glumes (CGL) } & Noires & 70,8 \\
& Marron clair & 8,3 \\
& Marron sombre & 10,4 \\
& Paille & 8,3 \\
& Jaune & 2,1 \\
\hline \multirow{2}{*}{ Vitrosité (VTR) } & Farineux & 16,7 \\
& Plutôt farineux & 8,3 \\
& Corné à 50\% & 31,2 \\
& Plutôt corné & 41,7 \\
& Vitreux & 2,1 \\
\hline \multirow{2}{*}{ Couleur des grains (CGL) } & Blanc ivoire & 20,8 \\
& Blanc mât & 52,1 \\
& Rouge & 6,2 \\
& Marron & 12,5 \\
& Brun & 6,2 \\
& Gris & 2,1 \\
\hline ART & Présent & 83,33 \\
Présence d'arêtes (ART) & Absent & 16,67 \\
\hline \multirow{2}{*}{ Compacité de la glume (CPA) } & Compacte & 8,33 \\
& Semi compacte & 79,17 \\
Pilosité de la glume & Lâche & 12,5 \\
\hline & Absent & 97,87 \\
& Présent & 2,13 \\
\hline
\end{tabular}

Tableau 4 : Matrice de corrélations entre les caractères étudiés.

\begin{tabular}{|c|c|c|c|c|c|c|c|c|c|c|c|c|c|c|}
\hline caractères & NJL & NJF & LOF & LAF & HPM & LOP & TAI & GPA & GPL & DTI & PCG & NEN & LAP & NTA \\
\hline NJF & 0,11 & 1 & & & & & & & & & & & & \\
\hline LOF & $-0,02$ & 0,60 & 1 & & & & & & & & & & & \\
\hline LAF & $-0,12$ & 0,21 & 0,34 & 1 & & & & & & & & & & \\
\hline HPM & 0,24 & $\mathbf{0 , 4 3}$ & 0,29 & $-0,31$ & 1 & & & & & & & & & \\
\hline LOP & 0,17 & $\mathbf{0 , 3 2}$ & 0,54 & 0,04 & 0,55 & 1 & & & & & & & & \\
\hline TAI & $-0,01$ & 0,20 & 0,12 & $-0,02$ & $-0,02$ & 0,06 & 1 & & & & & & & \\
\hline GPA & $-0,07$ & $-0,30$ & $-0,03$ & 0,26 & $-0,19$ & $-0,11$ & $-0,47$ & 1 & & & & & & \\
\hline GPL & $-0,15$ & $-0,18$ & 0,07 & 0,29 & $-0,07$ & 0,12 & $-0,12$ & $\mathbf{0 , 7 8}$ & 1 & & & & & \\
\hline DTI & $-0,04$ & 0,63 & 0,48 & 0,43 & 0,22 & 0,10 & 0,02 & $-0,02$ & $-0,04$ & 1 & & & & \\
\hline PCG & $-0,09$ & $-0,10$ & 0,01 & 0,16 & $-0,22$ & $-0,04$ & $-0,51$ & 0,11 & $-0,05$ & 0,02 & 1 & & & \\
\hline NEN & 0,07 & 0,69 & 0,42 & 0,24 & 0,35 & 0,03 & 0,12 & $-0,35$ & $-0,26$ & 0,76 & 0,05 & 1 & & \\
\hline LAP & $-0,06$ & $\mathbf{0 , 4 1}$ & 0,19 & 0,16 & $\mathbf{0 , 3 0}$ & 0,07 & $-0,06$ & $-0,03$ & $-0,03$ & $\mathbf{0 , 5 7}$ & $-0,29$ & $\mathbf{0 , 5 0}$ & 1 & \\
\hline NTA & 0,01 & 0,11 & 0,09 & $-0,04$ & $-0,08$ & 0,05 & $-0,01$ & $-0,23$ & $-0,23$ & $-0,16$ & 0,54 & 0,07 & $-0,37$ & 1 \\
\hline NTB & 0,07 & 0,28 & 0,25 & 0,08 & 0,18 & 0,27 & $\mathbf{0 , 7 7}$ & $-0,34$ & 0,13 & 0,06 & $-0,54$ & 0,22 & 0,05 & $-0,16$ \\
\hline
\end{tabular}




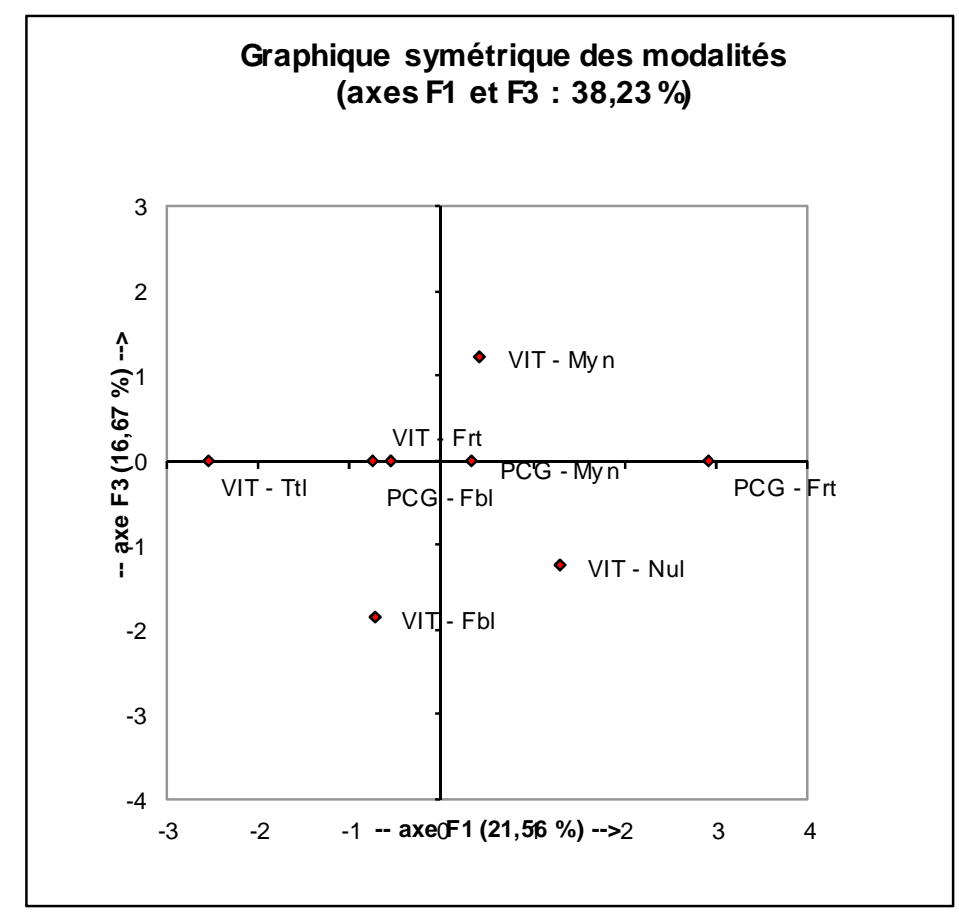

Figure 1: Modalité des variables sur l'axe 1×2 de l'ACM.

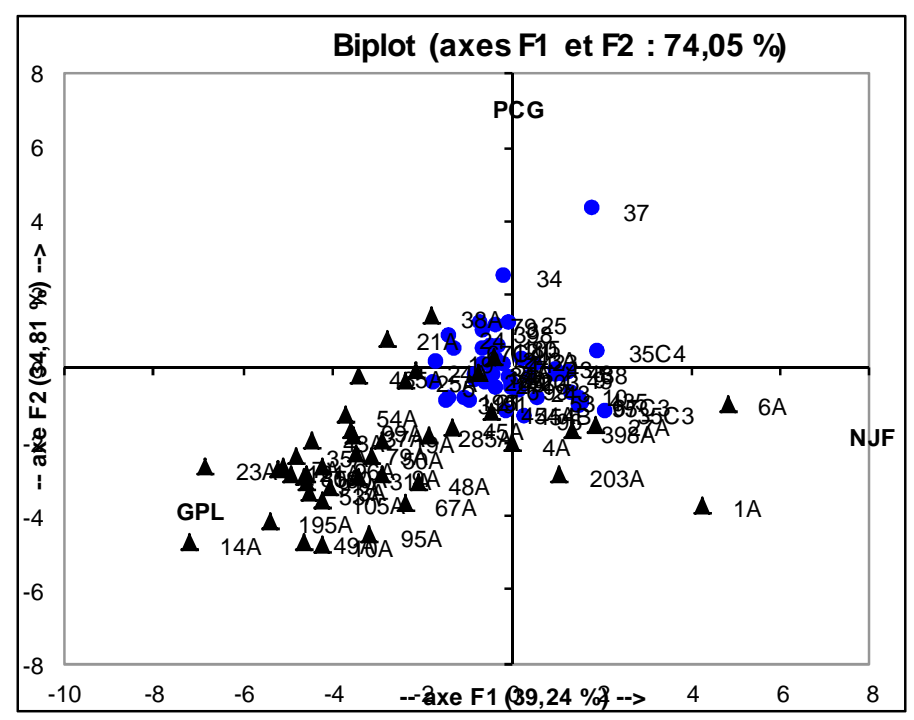

Figure 2 : Modalité des variables sur l'axe $1 \times 2$ de l'ACP. 


\section{DISCUSSION}

Les analyses de variance des caractères indiquent des différences significatives entre les écotypes pour la plupart de caractères sauf pour le poids de cent grains. Ce qui traduit l'existence d'une importante diversité agromorphologique. Les faibles valeurs des écart-types notées entre les performances des écotypes pour la plupart des caractères étudiés traduisent un regroupement des individus autour des valeurs moyennes. Ce qui ne masque toutefois pas une variabilité, notamment pour les caractères liés à la productivité.

La taille des plantes varie entre 107,7 et $395,8 \mathrm{~cm}$. Zongo (1991) et Barro/Kondombo et al. (2008) ont observé des hauteurs de plantes comprises respectivement entre 151 à $512 \mathrm{~cm}$ et 144 à $443 \mathrm{~cm}$. Le plus grand sorgho de l'échantillon est nettement plus petit que les plus grands écotypes de ces deux études. Il est justifié par l'objectif de sélection qui a orienté le choix vers les sorghos les plus performants. Vaksmann et al. (2005) ont rapporté que les sorghos de petite taille valorisent mieux les éléments fertilisants en diminuant la production de la paille au profit de la production grainière. Le cycle des écotypes varie entre 62 à 80 jours. Zongo (1991) a révélé des cycles allant de 58 à 135 jours. La valeur supérieure, 80 jours est inférieurs à 135 jours. Cet écart du cycle trouve son explication dans la corrélation négative qui existe entre le cycle et le rendement par plante. Ces résultats se rapprochent de ceux de Barro/Kondombo (2010) dont le cycle varie de 57 à 98 jours. Le choix des écotypes est en étroite relation avec la production, ce qui explique l'absence dans l'échantillon des écotypes de grandes tailles et à cycle long. Zongo (1991) a observé une forte corrélation positive entre le cycle et la hauteur de la plante. Cela contribue à expliquer l'absence de plantes de grande taille dans l'échantillon. Bezançon (2003) ajoute par ailleurs qu'il y a une relation linéaire entre la maturité et la hauteur des plantes, d'où la prépondérance des cultivars hâtifs plus courts en milieu paysans. $72,9 \%$ des écotypes sont blancs, ce groupe représentait $75,13 \%$ de l'échantillon national étudié par Zongo en 1991. Cela peut s'expliquer selon Trouche et al. (2008) par la forte demande des agriculteurs en sorgho blanc. Nebié et al. (2010-2012) rapportent quant à eux que 100\% des écotypes de sorgho à grains sucrés sont rouges. $43,75 \%$ de sorghos sont très vitreux avec plus de $90 \%$ qui sont blancs. $72,92 \%$ de sorgho sont vitreux et sont représentés à plus de $90 \%$ de sorghos blancs. Dje et al. (2006) ont trouvé que $60 \%$ de sorghos utilisés pour l'alimentation humaine au Maroc portent des grains vitreux. On observe également que $85,5 \%$ des sorghos sont aristés. Ces dernières sont des caractéristiques qui assurent une protection de la plante contre les oiseaux. Dje et al. (2006) ont trouvé que $95,2 \%$ des sorghos marocains portent des arêtes.

Parmi les corrélations observées, celles qui associent le rendement par plante au rendement paniculaire présentent un grand intérêt. Le rendement paniculaire contribue au rendement par plante. Le rendement par plante est également corrélé à la longueur et la largeur des feuilles. Les feuilles sont le siège de la photosynthèse. Elles permettent la fabrication des matières carbonées (glucides) stockées comme réserves dans le grain. Fiedel et al. (1983) rapportent que les glucides représentent $80 \%$ de la matière sèche du grain. Les meilleurs rendements paniculaires ne sont pas produits par des plantes de grandes tailles. Selon Vaksmann et al. (2008), la réduction de la taille de la variété locale permet l'amélioration des composantes du rendement. Les types durra et caudatum sont associés aux grains farineux. Ce qui corrobore les résultats des travaux de Zongo en 1991 qui rapporte que ce sont essentiellement les guinea qui sont vitreux alors que les autres races sont farineuses. Il existe une corrélation négative entre la vitrosité et la grosseur des grains. Les sorghos à gros grains sont totalement farineux et à l'opposé, ceux qui possèdent des petits grains sont vitreux. Chantereau et Nicou (1991) émettent l'hypothèse qu'il existerait 
une corrélation négative entre la grosseur des grains et la vitrosité. Les sorghos farineux ne possèdent pas d'arêtes, à l'opposé, les sorghos vitreux en possèdent. Ce qui laisse penser qu'il existerait une corrélation positive entre la vitrosité et l'aristation.

La différence qui ressort entre les deux évaluations avec des grandes valeurs portées par celle de 1991 pourrait avoir plusieurs raisons :

- Tout d'abord, les différents contextes dans les quels les travaux ont été menés. Le changement climatique qui a affecté notre planète entraînant avec lui la raréfaction de la pluie le long du gradient nord-sud des isohyètes semble expliquer de cette différence. Traore et al. (2000) qui ont travaillé sur l'adaptation à la sécheresse des écotypes locaux de sorghos au Mali ont montré que malgré la baisse pluviométrique observée au cours des 40 années d'études, il n'y a pas eu de changements significatifs des dates de début et de fin de la saison pluvieuse. Bezançon (2003) qui a travaillé sur l'évolution de la diversité des mils et sorghos cultivés au Niger entre 1976 et 2003 a observé des changements significatifs pour certaines caractéristiques morphologiques ou phénologiques. Il précise que la floraison est plus précoce et les plantes plus petites en 2003 qu'en 1976 pour le mil et le sorgho. Selon Kouressy et al. (2008), en 20 ans, le cycle moyen des cultivars locaux s'est raccourci de 5 jours. Ce qui pourrait expliquer les différences de taille et de cycle semi-épiaison des écotypes entre l'évaluation 2008 et celle de 1991. Selon les mêmes auteurs, cette différence correspondrait bien à une adaptation rapide aux changements climatiques dans le contexte d'une succession d'années plus sèches.

- La photosensibilité permet aux sorghos de faire face aux incertitudes climatiques en modulant la durée de leur cycle de façon à ce que la floraison reste groupée à la fin de la saison des pluies (Vaksmann et al., 1996). Cette sensibilité à la photopériode est une caractéristique propre aux sorghos ouest- africains (Vaksann et al., 2008). L'évaluation menée pendant la campagne agricole 2008 par rapport à celle de 1991 a accusée un retard de semi de 23 jours. Ce qui pourrait expliquer la chute de rendement car selon Touré et al. (1998), les sorghos sensibles à la photopériode tendent de réduire leur nombre de grains par panicule de l'ordre de $34 \%$ à $58 \%$ suite à un retard de semis de 15 à 30 jours

- La petite taille des plantes, le faible tallage et le faible nombre de nœuds de l'évaluation 2008 sont la conséquence directe de la réduction du cycle précédemment mentionnée. Ce qui, selon Traore et al. (2000) est une caractéristique propre aux plantes photopériodiques. Celle-ci permet aux sorghos de produire, quelle que soit la variation de la saison, en interrompant le développement végétatif au profit de la production grainière.

\section{Conclusion}

Au terme de cette étude, retenons que la caractérisation agromorphologique a permis de montrer l'existence d'une importante variabilité au sein des écotypes performants de la collection autours des caractères liés à la production. Quatre des cinq races de sorghos sont présentes (seule la race kaffir n'y est pas). La plupart des sorghos sont à cycle court et possèdent des grains vitreux et blancs. Il en ressort également des associations dont les plus intéressantes sont celles qui d'une part associent le cycle de la plante à la taille et au tallage, le rendement par panicule au rendement par plante, et d'autre part opposent le tallage au poids de la panicule principale et au poids de cent grains. Il en ressort également deux corrélations dont l'une positive entre la vitrosité et la présence d'arêtes et une autre négative entre la vitrosité et la grosseur de la graine.

Le choix des meilleurs écotypes a permis de retenir quatre écotypes dont la vulgarisation pourrait être envisagée pour améliorer la productivité et la qualité des sorghos burkinabé. L'identification des géniteurs a en l'occurrence, permis de déterminer quelques sorghos porteurs de 
caractères dont l'utilisation comme géniteurs pourraient éventuellement permettre l'amélioration des sorghos.

\section{REFERENCES}

Barro/Kondombo CP, Broke KV, Chantereau J, Sagnard F, Zongo JD. 2008. Variabilité phénotypique des sorghos locaux de deux régions du Burkina Faso: la Boucle du Mouhoun et le Centre-Ouest. Cahiers d'Agricultures, 17(2) : 107-113.

Barro/Kondombo CP. 2010. Diversité agromorphologique et génétique de variétés locales de sorgho (Sorghum bicolor [L.] Moench) au Burkina Faso. Eléments pour la valorisation des ressources génétiques locales. Thèse de doctorat, Université de Ouagadougou, $112 \mathrm{p}$.

Bezançon G. 2003. Evolution de la diversité des mils et sorghos cultivés au Niger entre 1976 et 2003: Influence des facteurs naturels et anthropiques. Diversité et Génomes des Plantes Cultivés, 5p.

Brocke KV, Trouche G, Vaksmann M, Bazile D. 2002. Préservation de l'agrobiodiversité du sorgho au Mali et au Burkina Aso -amélioration et la productivité et maintien de la biodiversité. Atelier sur la diversité et valorisation des ressources génétiques des mils. Niamey (Niger) 28-29 mai 2002, Cirad, 9p.

Chantereau J, Nicou R. 1991. Le Sorgho. ACCT : Paris ; 159.

Chantereau J, Trouche G, Luce C, Deu M, Hamon P. 1997. Le sorgho. In L'Amélioration des Plantes Tropicales, Charrier A, Jacquot M, Hamon S, Nicolas D (eds). Edit Cirad, Collection Repères : Paris ; 565-590.

Dje Y, Heuertz M, Ater M, Lefebvre C, Vekmans X. 2006. Evaluation de la Diversité Morphologique des Variétés de sorgho du Nord-Ouest du Maroc. Jr. Biotechnol. Agrom. Soc. Environ., 2(1): 39-46.
Fiedel G, Marti A, Thiebaut S. 1983. Caractérisation et valorisation du sorgho. CIRAD-ca, 349p.

Hanak-Freud H. 2000. Au Burkina Faso, les céréales traditionnelles peuvent devenir des cultures de rente. Agriculture et Développement, 23: 18-30.

Kouressy M, Traore S, Vaksmann M, Grum M, Maikano I, Soumare M, Traore PS, Bazile D, Dingkuhn M, sidibe A. 2008. Adaptation des sorghos du Mali à la variabilité climatique. Cahiers Agricultures, 17(2), mars-avril 2008.

Nebié B, Gapili N, Traoré RE, Nanema KR, Bationo/Kando P, Sawadogo M, Zongo JD. 2010-2012. Diversité phénotypique des sorghos à grains sucrés du centre nord du Burkina Faso. Sciences et Techniques, Sciences Naturelles et Agronomie, 32(12): 2010-2012.

Nebié B, Nanema KR, Bationo/Kando P, Traoré RE, Labeyrie V, Sawadogo N, Sawadogo M, Zongo JD. 2013. Variation des caractères agromorphologiques et du brix d'une collection de sorghos à tige sucrée du Burkina Faso. Int. J. Biol. Chem. Sci., 7(5): 1919-1928.

Traore SB, Reyniers FN, Vaksmann M, Kone B, Sidibe A, Yorote A, Yattara K, Kouressey M. 2000. Adaptation à la sécheresse des écotypes locaux de sorghos du Mali. Science et Changements Planétaires/Sécheresse, 11(4): 227-237.

Trouche G, Da S, Pale G, Sohoro A, Ouedraogo O, Den G. 2001. Evaluation participative de nouvelles variétés de sorgho au Burkina. In Sélection Participative, Hocdé H, Lançon J, Trouche G (eds). Cirad-INERA : Montpellier ; 36-55.

Trouche G, Acuna SA, Hocde H, Solis RO, Zildhean CW, 2008. Valorisation de la diversité génétique du sorgho par des approches de sélection participative au Nicaragua. Cahiers d'Etudes et de Recherches Francophones/Agricultures. 17(2): 154-159. 
Toure A, Traore K, Bengaly A, Scheuring JF, Rosenow DT, Rooney LW. 1998. The potential of local cultivars in sorghum improvement in Mali. J. African Crop Sc., 6(1): 1-7.

Vaksmann M, Traore SB, Niangado O. 1996. Le photopériodisme des sorghos africains. Agriculture et Développement, 9: $13-8$.

Vaksmann M, Kouressey M, Toure A. 2005. Valorisation de la diversité génétique des sorghos en zone cotonnière du Mali grâce à la sélection décentralisée et participative. In Partenaires pour Construire des Projets de Sélection Participative, Lancon J, Floquet A, Weltzien E. Actes de l'atelier-recherche, 14-18 mars 2005, Cotonou, Benin. Montpelier : CIRAD : éditions, INRAB, Coopération française, 2006.
Vaksmann M, Kouressey M, Chantereau J, Bazile D, Sangard F, Toure A, Sanogo O, Diawara G, Dante A. 2008. Utilisation de la diversité génétique des sorghos locaux $\mathrm{du}$ Mali. Cahiers d'Etudes et de Recherches Francophones/ Agricultures. 17( 2): 140-145.

Zongo JD. 1991. Ressources génétiques des sorghos (Sorghum bicolor (L.) Moench) du Burkina Aso: Evaluation agromorphologique et génétique. Thèse de docteur ès science, sciences naturelles, Université d'Abidjan, Côte d'Ivoire, 219p. 